\title{
Dirty necrosis
}

National Cancer Institute ( $\mathrm{NCl})$

\section{Source}

National Cancer Institute (NCI). Dirty necrosis.

Presence of necrotic cellular debris within the lumen of the neoplastic glands in the colorectal mucosa. 